\title{
Metrical oxidation states of 1,4-diazadiene (DAD)
}

\section{derived ligands}

Felix J. de Zwart, ${ }^{a}$ Bente Reus, ${ }^{a}$ Annechien A.H.L. Laporte, ${ }^{a}$ Vivek Sinha ${ }^{b}$ and Bas de Bruin ${ }^{a *}$

${ }^{a} H o m o g e n e o u s$, Supramolecular and Bio-Inspired Catalysis, Van 't Hoff Institute for

Molecular Sciences, University of Amsterdam, 1098 XH Amsterdam, The Netherlands.

${ }^{\mathrm{b}}$ Inorganic Systems Engineering, Department of Chemical Engineering, Delft University of Technology, Van der Maasweg 9, Delft 2629 HZ, Netherlands.

E-mail: b.debruin@uva.nl 


\section{Contents}

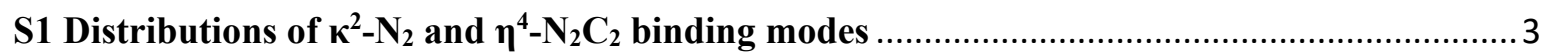

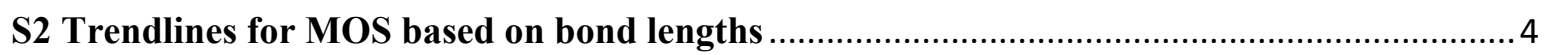

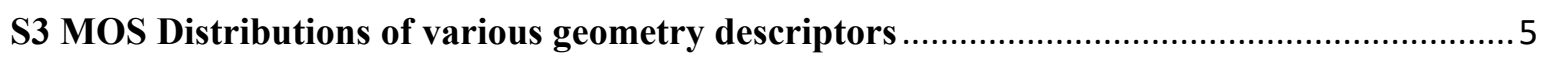

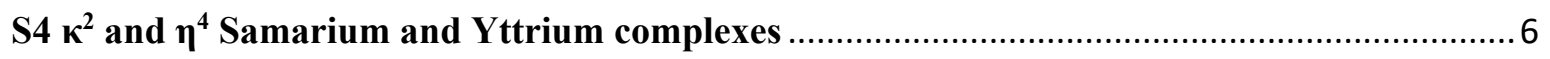

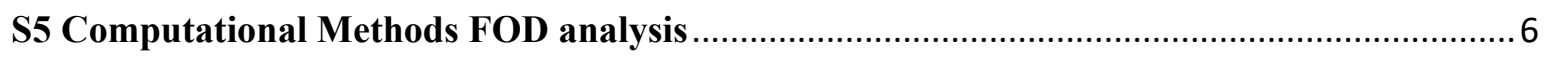

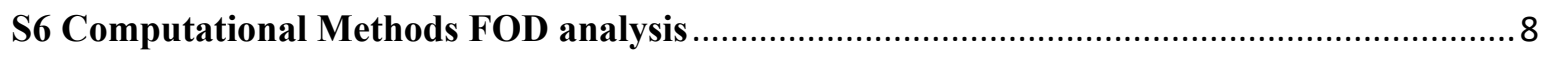

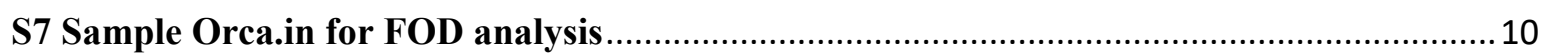

S8 Full results of the FOD analysis of [M(trop 2 dad)] complexes............................................... 11

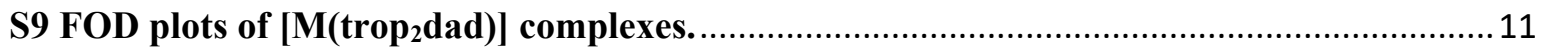




\section{S1 Distributions of $\kappa^{2}-\mathrm{N}_{2}$ and $\eta^{4}-\mathrm{N}_{2} \mathrm{C}_{2}$ binding modes}
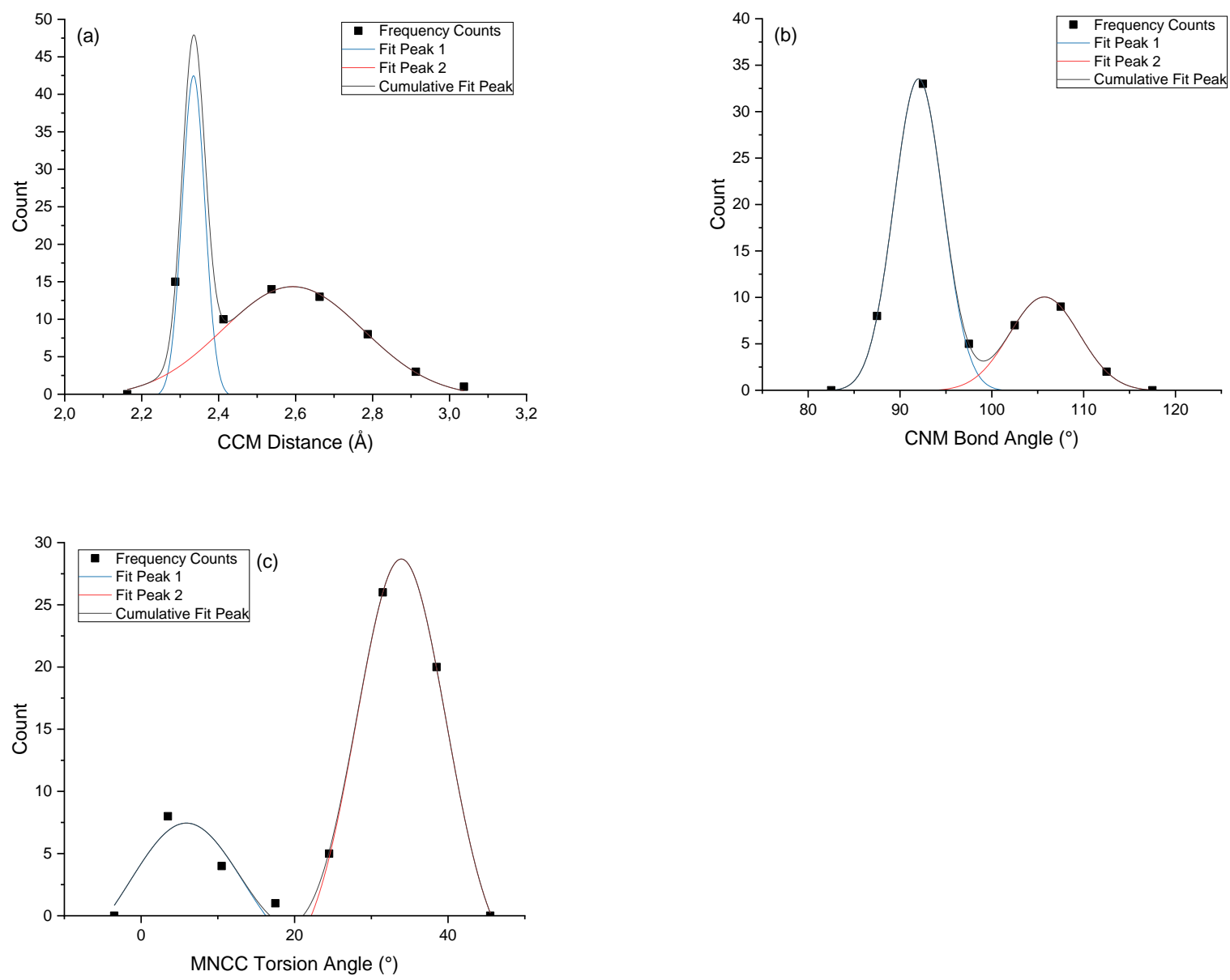

Figure S1. Observed Gaussian Distribution of the geometric parameters after frequency counts: (a) $\mathrm{C}=\mathrm{C} \rightarrow \mathrm{M}$ distance; (b) $\mathrm{C}-\mathrm{N}-\mathrm{M}$ bond angle; (c) $\mathrm{M}-\mathrm{N}-\mathrm{C}-\mathrm{C}$ torsion angle. 


\section{S2 Trendlines for MOS based on bond lengths}

Table S1. Trend between the average bond lengths and the ligand oxidation state for the two binding modes using the $\mathrm{C}=\mathrm{C} \rightarrow \mathrm{M}$ distance as descriptor $\left(\mathrm{y}=\mathrm{a}+\mathrm{bx}+\mathrm{cx}^{2}\right)$.

\begin{tabular}{|l|l|l|l|l|}
\hline $\mathbf{C C M}$ & Bond & $\mathbf{a}$ & $\mathbf{b x}$ & $\mathbf{c x}^{2}$ \\
\hline Coordinated $\left(\eta^{4}\right)$ & C-N & 1.2858 & -0.0553 & \\
\hline & C-C & 1.4932 & 0.115 & 0.0278 \\
\hline $\begin{array}{l}\text { Uncoordinated } \\
\left(\kappa^{2}\right)\end{array}$ & C-N & 1.2822 & -0.066 & \\
\hline & C-C & 1.4932 & 0.1087 & 0.0215 \\
\hline
\end{tabular}

Table S2. Trend between the average bond lengths and the ligand oxidation state for the two binding modes using the $\mathrm{M}-\mathrm{N}-\mathrm{C}$ bond angle as descriptor $\left(\mathrm{y}=\mathrm{a}+\mathrm{bx}+\mathrm{cx}^{2}\right)$.

\begin{tabular}{|l|l|l|l|l|}
\hline MNC & Bond & $\mathbf{a}$ & $\mathbf{b x}$ & $\mathbf{c x}^{2}$ \\
\hline Coordinated $\left(\eta^{4}\right)$ & $\mathrm{C}-\mathrm{N}$ & 1.2842 & -0.0599 & \\
\hline & $\mathrm{C}-\mathrm{C}$ & 1.4932 & 0.1134 & 0.0262 \\
\hline $\begin{array}{l}\text { Uncoordinated } \\
\left(\kappa^{2}\right)\end{array}$ & $\mathrm{C}-\mathrm{N}$ & 1.2821 & -0.0663 & \\
\hline & $\mathrm{C}-\mathrm{C}$ & 1.4932 & 0.1058 & 0.0186 \\
\hline
\end{tabular}

Table S3. Trend between the average bond lengths and the ligand oxidation state for the two binding modes using the $\mathrm{M}-\mathrm{N}-\mathrm{C}-\mathrm{C}$ torsion angle as descriptor $\left(\mathrm{y}=\mathrm{a}+\mathrm{bx}+\mathrm{cx}^{2}\right)$.

\begin{tabular}{|l|l|l|l|l|}
\hline MNCC & Bond & $\mathbf{a}$ & $\mathbf{b x}$ & $\mathbf{c x}^{2}$ \\
\hline Coordinated $\left(\eta^{4}\right)$ & C-N & 1.2839 & -0.0608 & \\
\hline & C-C & 1.4932 & 0.1129 & 0.0257 \\
\hline $\begin{array}{l}\text { Uncoordinated } \\
\left(\kappa^{2}\right)\end{array}$ & C-N & 1.2823 & -0.0656 & \\
\hline & C-C & 1.4932 & 0.1043 & 0.0171 \\
\hline
\end{tabular}




\section{S3 MOS Distributions of various geometry descriptors}

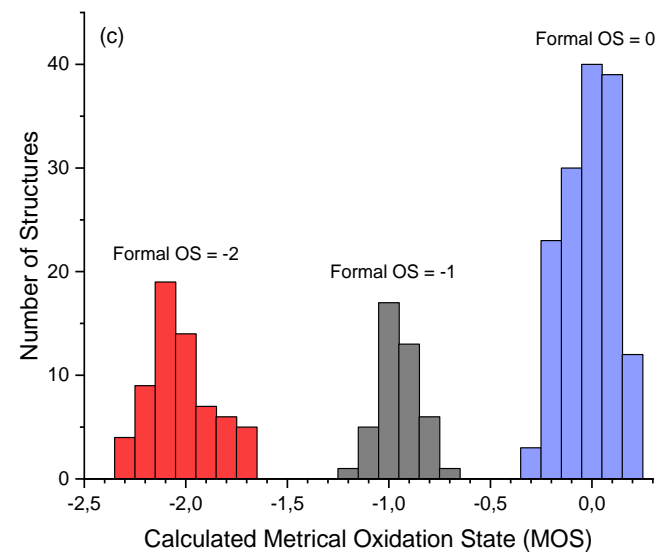

Figure S2. MOS distributions for the model using $\mathrm{C}=\mathrm{C} \rightarrow \mathrm{M}$ distance as descriptor.

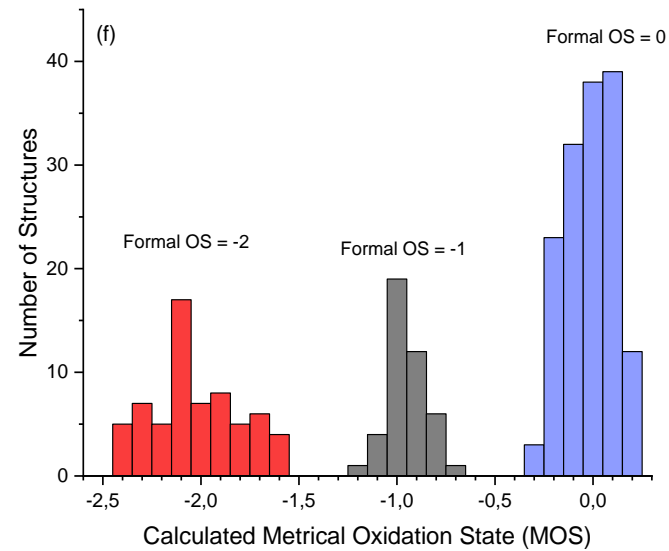

Figure S3. MOS distributions for the model using $\mathrm{M}-\mathrm{N}-\mathrm{C}$ angle as descriptor.

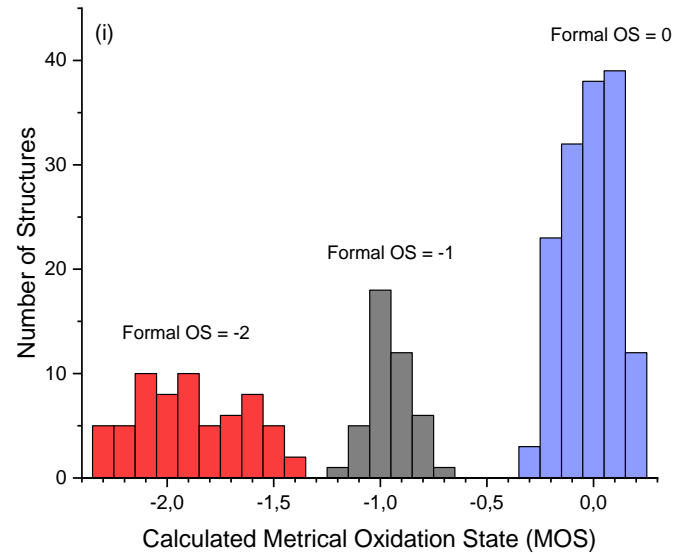

Figure S4. MOS distributions for the model using $\mathrm{M}-\mathrm{N}-\mathrm{C}-\mathrm{C}$ torsion angle as descriptor. 


\section{S4 $\kappa^{2}$ and $\eta^{4}$ Samarium and Yttrium complexes}

Table S4. Samarium and Yttrium complexes that were described as $\kappa^{2}$ binding mode by $\mathrm{C}=\mathrm{C} \rightarrow \mathrm{M}$ distance but $\eta^{4}$ by torsion angle.

\begin{tabular}{|l|l|l|l|l|l|l|}
\hline Refcode & CCM & MNCC & C-N $(\AA)$ & C-C $(\AA)$ & Ligand OS & Metal \\
\hline HAKWEX & 2.631 & 34.174 & 1.4105 & 1.358 & -2 & Sm(III) \\
\hline HAKWIB & 2.629 & 36.6165 & 1.425 & 1.377 & -2 & Sm(III) \\
\hline UWOBUF & 2.736 & 32.8495 & 1.4225 & 1.373 & -2 & Sm(III) \\
\hline DUDRAW & 2.614 & 36.207 & 1.425 & 1.376 & -2 & Y(III) \\
\hline DUDREA & 2.495 & 38.479 & 1.4235 & 1.377 & -2 & Y(III) \\
\hline HUBHUJ & 2.774 & 26.0025 & 1.423 & 1.362 & -2 & Y(III) \\
\hline HUBJAR & 2.638 & 34.68 & 1.4285 & 1.373 & -2 & Y(III) \\
\hline HUBJAR & 2.611 & 35.28 & 1.4315 & 1.371 & -2 & Y(III) \\
\hline HUBJEV & 2.763 & 26.186 & 1.4215 & 1.363 & -2 & Y(III) \\
\hline HUBJIZ & 2.808 & 29.131 & 1.4195 & 1.363 & -2 & Y(III) \\
\hline NOJKAB & 2.580 & 36.2945 & 1.423 & 1.381 & -2 & Y(III) \\
\hline NOJKEF & 2.678 & 31.1115 & 1.4205 & 1.386 & -2 & Y(III) \\
\hline NOJKEF & 2.599 & 34.5735 & 1.4135 & 1.367 & -2 & Y(III) \\
\hline NOJKIJ & 2.630 & 32.939 & 1.426 & 1.365 & -2 & Y(III) \\
\hline NOJKIJ & 2.653 & 31.67 & 1.4315 & 1.336 & -2 & Y(III) \\
\hline BUDZOR & 2.540 & 39.359 & 1.408 & 1.358 & -2 & Y(III) \\
\hline FAKZAU & 2.587 & 36.2305 & 1.4085 & 1.364 & -2 & Y(III) \\
\hline LAYFID & 2.527 & 34.5455 & 1.4215 & 1.354 & -2 & Y(III) \\
\hline LECZIF & 2.578 & 35.2735 & 1.402 & 1.362 & -2 & Y(III) \\
\hline LECZIF & 2.582 & 34.734 & 1.4125 & 1.352 & -2 & Y(III) \\
\hline
\end{tabular}

S5 Aluminium DAD complexes MOS analysis

\begin{tabular}{|l|r|r|r|r|r|}
\hline Refcode & $\mathrm{C}-\mathrm{N}(\AA)$ & $\mathrm{C}-\mathrm{C}(\AA)$ & $\mathrm{CCM}(\AA)$ & MOS & esd \\
\hline HUKNUY & 1.455 & 1.341 & 2.543 & -2.66 & 0.14 \\
\hline PIGSAB & 1.447 & 1.324 & 2.547 & -2.54 & 0.26 \\
\hline GUGCIW & 1.440 & 1.351 & 2.508 & -2.48 & 0.08 \\
\hline HUJYAO & 1.440 & 1.346 & 2.508 & -2.48 & 0.12 \\
\hline YOQPIG & 1.443 & 1.333 & 2.599 & -2.45 & 0.17 \\
\hline JODZIN & 1.439 & 1.341 & 2.554 & -2.43 & 0.13 \\
\hline SESDOL & 1.438 & 1.334 & 2.582 & -2.40 & 0.17 \\
\hline HUKNEI & 1.434 & 1.344 & 2.507 & -2.40 & 0.13 \\
\hline HUKNAE & 1.435 & 1.349 & 2.549 & -2.36 & 0.08 \\
\hline PIGRUU & 1.433 & 1.335 & 2.526 & -2.36 & 0.19 \\
\hline PIGSAB & 1.434 & 1.335 & 2.547 & -2.36 & 0.18 \\
\hline BAXVAZ & 1.430 & 1.336 & 2.498 & -2.35 & 0.20 \\
\hline PIGRUU & 1.432 & 1.345 & 2.526 & -2.34 & 0.12 \\
\hline HUKNIM & 1.430 & 1.347 & 2.507 & -2.34 & 0.11 \\
\hline
\end{tabular}


Supporting Information

\begin{tabular}{|c|c|c|c|c|c|}
\hline BAXTEB & 1.433 & 1.334 & 2.583 & -2.33 & 0.18 \\
\hline PIGROO & 1.432 & 1.344 & 2.546 & -2.33 & 0.12 \\
\hline YOQPOM & 1.433 & 1.349 & 2.593 & -2.32 & 0.07 \\
\hline YOQPOM & 1.433 & 1.35 & 2.599 & -2.31 & 0.06 \\
\hline HUJZIX & 1.428 & 1.336 & 2.517 & -2.30 & 0.19 \\
\hline HUKMUX & 1.428 & 1.343 & 2.517 & -2.29 & 0.14 \\
\hline YOQPUS & 1.431 & 1.343 & 2.593 & -2.29 & 0.11 \\
\hline BAXVAZ & 1.426 & 1.345 & 2.498 & -2.29 & 0.13 \\
\hline YOQPUS & 1.429 & 1.34 & 2.573 & -2.27 & 0.14 \\
\hline HUKPAG & 1.426 & 1.342 & 2.543 & -2.25 & 0.14 \\
\hline HUKPAG & 1.425 & 1.343 & 2.536 & -2.24 & 0.13 \\
\hline HUKNOS & 1.425 & 1.353 & 2.555 & -2.21 & 0.05 \\
\hline HUKMUX & 1.422 & 1.335 & 2.549 & -2.20 & 0.19 \\
\hline HUKNOS & 1.421 & 1.356 & 2.555 & -2.15 & 0.04 \\
\hline VICSOP & 1.414 & 1.311 & 2.499 & -2.15 & 0.39 \\
\hline ZIWDUE & 1.413 & 1.337 & 2.499 & -2.11 & 0.20 \\
\hline VICSIJ & 1.413 & 1.342 & 2.585 & -2.05 & 0.15 \\
\hline PUNROF & 1.406 & 1.33 & 2.514 & -2.02 & 0.26 \\
\hline PIGREE & 1.410 & 1.349 & 2.546 & -2.02 & 0.11 \\
\hline PIFDEP & 1.382 & 1.391 & 2.57 & -1.48 & 0.09 \\
\hline BAXTIF & 1.360 & 1.399 & 2.583 & -1.17 & 0.03 \\
\hline JODZOT01 & 1.355 & 1.406 & 2.57 & -1.08 & 0.04 \\
\hline VICSIJ & 1.335 & 1.391 & 2.585 & -0.95 & 0.16 \\
\hline BAXTOL & 1.352 & 1.426 & 2.536 & -0.94 & 0.15 \\
\hline BAXTUR & 1.353 & 1.434 & 2.518 & -0.91 & 0.20 \\
\hline LUHWUG & 1.337 & 1.409 & 2.568 & -0.88 & 0.05 \\
\hline BAXTUR & 1.351 & 1.436 & 2.518 & -0.88 & 0.20 \\
\hline JODZEJ & 1.352 & 1.438 & 2.536 & -0.87 & 0.21 \\
\hline BAXTOL & 1.345 & 1.445 & 2.536 & -0.76 & 0.21 \\
\hline JODZOT & 1.346 & 1.466 & 2.554 & -0.65 & 0.33 \\
\hline LUHXAN & 1.308 & 1.442 & 2.568 & -0.45 & 0.06 \\
\hline CIQSUS & 1.282 & 1.46 & 2.573 & -0.17 & 0.16 \\
\hline MIMYEN & 1.268 & 1.46 & 2.514 & -0.08 & 0.26 \\
\hline XIKLEL & 1.283 & 1.496 & 2.598 & 0.01 & 0.02 \\
\hline XIKLAH & 1.288 & 1.502 & 2.603 & 0.01 & 0.08 \\
\hline RUPFAL & 1.288 & 1.506 & 2.618 & 0.03 & 0.10 \\
\hline SEZGAI & 1.287 & 1.504 & 2.582 & 0.03 & 0.08 \\
\hline RUPFAL & 1.287 & 1.505 & 2.604 & 0.03 & 0.08 \\
\hline XIKKUA & 1.289 & 1.507 & 2.581 & 0.04 & 0.10 \\
\hline RUPFAL & 1.286 & 1.504 & 2.604 & 0.04 & 0.07 \\
\hline SEZGEM & 1.287 & 1.507 & 2.557 & 0.05 & 0.09 \\
\hline XIKLAH & 1.284 & 1.506 & 2.603 & 0.06 & 0.07 \\
\hline RUPFAL & 1.283 & 1.506 & 2.618 & 0.06 & 0.06 \\
\hline XIKLEL & 1.281 & 1.505 & 2.598 & 0.08 & 0.04 \\
\hline SEZGEM & 1.281 & 1.505 & 2.557 & 0.08 & 0.03 \\
\hline
\end{tabular}


S6 N-cyclohexyl DAD complexes MOS analysis

\begin{tabular}{|l|l|l|l|l|l|l|}
\hline Metal & Refcode & $\mathrm{C}-\mathrm{N}(\AA)$ & $\mathrm{C}-\mathrm{C}(\AA)$ & $\mathrm{CCM}(\AA)$ & MOS & esd \\
\hline $\mathrm{Cr}(\mathrm{III})$ & NINGEY & 1.3315 & 1.389 & 2.766 & -0.92 & 0.201 \\
\hline $\mathrm{Ni}(\mathrm{II})$ & TAYDUV & 1.3415 & 1.426 & 2.674 & -0.83 & 0.076 \\
\hline $\mathrm{Ni}(\mathrm{II})$ & TAYDUV & 1.3375 & 1.423 & 2.668 & -0.81 & 0.033 \\
\hline $\mathrm{Ru}(\mathrm{II})$ & MEZJUZ & 1.2735 & 1.436 & 2.771 & -0.24 & 0.327 \\
\hline $\mathrm{Cu}(\mathrm{I})$ & IXANIG & 1.28 & 1.46 & 2.724 & -0.16 & 0.167 \\
\hline $\mathrm{Cu}(\mathrm{I})$ & IXAMOL & 1.279 & 1.467 & 2.652 & -0.12 & 0.141 \\
\hline $\mathrm{Cu}(\mathrm{I})$ & IXAMOL & 1.273 & 1.461 & 2.644 & -0.11 & 0.211 \\
\hline $\mathrm{Cu}(\mathrm{I})$ & IXAMOL & 1.2705 & 1.46 & 2.648 & -0.10 & 0.234 \\
\hline $\mathrm{Pd}(\mathrm{II})$ & PIJMOL & 1.2775 & 1.47 & 2.812 & -0.09 & 0.138 \\
\hline $\mathrm{Fe}(\mathrm{II})$ & ISIBIW & 1.263 & 1.472 & 2.736 & 0.01 & 0.231 \\
\hline $\mathrm{Mo}(\mathrm{II})$ & DADGAQ & 1.258 & 1.467 & 2.902 & 0.02 & 0.289 \\
\hline $\mathrm{Fe}(\mathrm{II})$ & KOMWAL & 1.2685 & 1.48 & 2.813 & 0.02 & 0.156 \\
\hline $\mathrm{Pd}(\mathrm{II})$ & LUQXAX & 1.2825 & 1.497 & 2.715 & 0.02 & 0.019 \\
\hline $\mathrm{Fe}(\mathrm{II})$ & MUDVAI & 1.238 & 1.496 & 2.741 & 0.27 & 0.304 \\
\hline
\end{tabular}

\section{S7 Computational Methods FOD analysis}

The most reliable way to probe multi-reference character in metal complexes is to use multiconfigurational post-Hartree-Fock methods such as complete active space self-consistent field CASSCF calculations. ${ }^{1}$ However, such calculations are time-consuming, labor-intensive, errorprone to set-up and often require prior knowledge of the electronic structure. As such, we decided to combine the MOS calculator with an alternative and easier to use computational tool to gain further insight into the electronic structures of these $\left[\mathrm{M}\left(\operatorname{trop}_{2} \mathrm{dad}\right)\right]$ complexes: Fractional Occupation Density (FOD) analysis. We used these FOD calculations to distinguish between localized and delocalized situations of metal-ligand $\pi$-electrons in [M(trop 2 dad)] complexes $(\mathrm{M}=\mathrm{Fe}, \mathrm{Ru}, \mathrm{Rh})$ in the singlet spin state $(\mathrm{S}=0)$ as discussed in the main text. The FOD analysis yields NFOD values and plots of 'hot electron' distributions based on FOD. Generally, we consider (electronic singlet) complexes with $\mathrm{NFOD}_{\mathrm{FO}}>1$ values and DAD $\pi$-type 'hot electrons' in the FOD plots to have substantial $\pi$-electron delocalization contributions, with a significant likelihood of multi-reference contributions to the ground state wavefunction. 


\section{Supporting Information}

The fractional occupation density (FOD) calculations were carried out using Orca 4.1.2., ${ }^{2,3}$ They were used to obtain $\mathrm{N}_{\mathrm{FOD}}$ values and 'hot electron' density plots based on FOD. NFOD is obtained by spatial integration of the $\rho^{F O D}(r)$ given by:

$$
\begin{gathered}
(2) \rho^{F O D}(r)=\sum_{i}^{N}\left(\delta_{1}-\delta_{2} f_{i}\right)\left|\phi_{i}(r)\right|^{2} \\
f_{i}=\frac{1}{e^{\left(\epsilon_{i}-E_{f}\right) / k T}+1}, k=\text { Boltzman constant }, T=\text { electronic temperature } \\
\delta_{1}=\delta_{2}=1, \epsilon_{i}<E_{f}, E_{f}=\text { Fermi energy level } \\
\delta_{1}=0, \delta_{2}=-1, \epsilon_{i}>E_{f}
\end{gathered}
$$

In equation (2) the summation runs over all electronic single-particle energy levels in the system and $\phi_{i}(r)$ are molecular spin orbitals. The FOD analysis was carried out using the standard settings in Orca invoked via the !FOD keyword (TPSS ${ }^{4} \mathrm{XC}$, def2-TZVP ${ }^{5}$ basis set, T $=5000 \mathrm{~K}){ }^{6}$ Large gap insulators such as alkanes have a very small $\mathrm{N}_{\mathrm{FOD}}<0.001$. NFOD increases for systems with double-bonds and for example reaches a value of 0.2 for octatetraene, due to the decreased HOMO-LUMO gap. ${ }^{3}$ Grimme and co-workers ${ }^{6}$ demonstrated that $\mathrm{N}_{\mathrm{FOD}}$ correlates well with the computed biradical character (y), which is given by:

$$
\text { (3) } y=1-\frac{4\left(n_{\text {Hомо }}-n_{\text {LUMO }}\right)}{4+\left(n_{\text {Hомо }}-n_{\text {LUMO }}\right)^{2}}
$$

Here nHOMO and nLUMo represent the occupation number for HOMO and LUMO orbitals. For a perfect biradical, $\mathrm{n}_{\text {Hомо }}=\mathrm{n}_{\text {LUMO, }}$, and $\mathrm{y}=1$, whereas a biradical character of 0.63 corresponded to an $\mathrm{N}_{\mathrm{FOD}} \sim 1.77 .^{6}$ In an ethylene molecule with a $\mathrm{H}-\mathrm{C}=\mathrm{C}-\mathrm{H}$ torsion angle of $90^{\circ}$ the $\mathrm{N}_{\mathrm{FOD}}=2.0$ which corresponds to a perfect biradical. For comparison, we also performed the FOD analysis for ${ }^{1} \mathrm{O}_{2}$ which resulted in a $\mathrm{N}_{\mathrm{FOD}}=2.00$ indicating formation of a perfect biradical. 


\title{
Supporting Information
}

We performed FOD analysis on the $\left[\mathrm{M}\left(\right.\right.$ trop $_{2}$ dad $\left.)\right]$ complexes using the $\mathrm{x}$-ray structures from which we manually removed the counterions. Such a comparison allows us to separate static electron correlation effects arising from the multireference character due to the M-DAD bonding and those due to the counterion. FOD calculations lead to fractionally occupied molecular orbitals and the corresponding electronic density plot (FOD plot) confirmed that the 'hot electrons' are largely involved in metal-DAD $\pi$-interaction orbitals. We use NFOD to identify complexes with a high potential for multireference character, indicative for substantial $\pi$-delocalization, metal-to-ligand (single) electron transfer and/or biradical character. For reference, the $\mathrm{Ru}(\mathrm{PNP})$ pincer complex $\left[\mathrm{Ru}^{\mathrm{II}}(\mathrm{CO})(\mathrm{H})(\mathrm{PNP})\right]\left(\right.$ where $\mathrm{PNP}=\mathrm{P}\left({ }^{\mathrm{i}} \mathrm{Pr}\right)_{2}-\left(\mathrm{CH}_{2}\right)_{2}-\mathrm{N}-$ $\left.\left(\mathrm{CH}_{2}\right)_{2}-\mathrm{P}\left({ }^{\mathrm{i}} \mathrm{Pr}\right)_{2}\right)^{7}$, which is not expected to have any significant multi-reference character, was found to have a small $\mathrm{N}_{\mathrm{FOD}}=0.20$ values in such FOD calculations.

\section{S8 Sample Orca.in for FOD analysis}

\author{
Sample ${ }^{1} \mathrm{O}_{2}$ Orca.in for FOD analysis
}

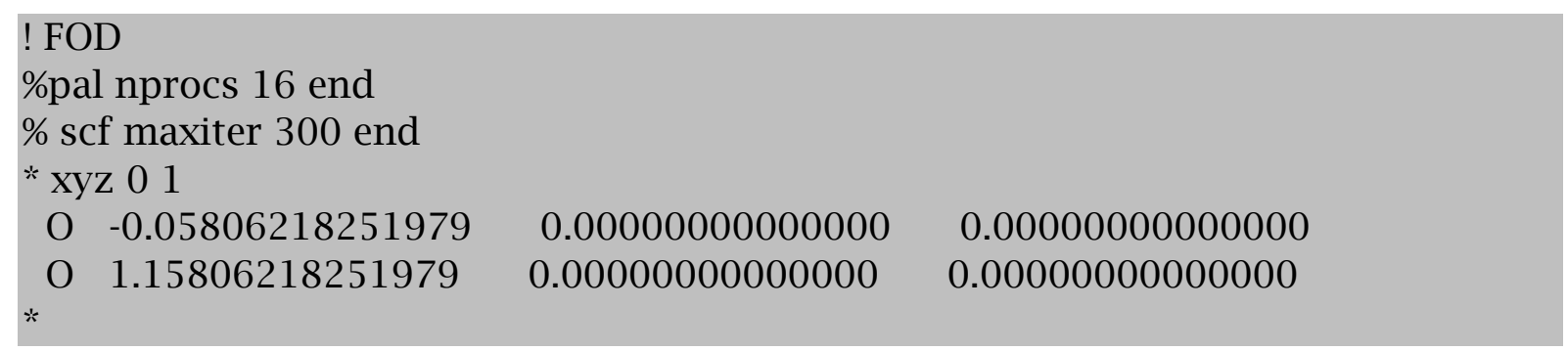




\section{S9 Full results of the FOD analysis of $\left[\mathrm{M}\left(\operatorname{trop}_{2} \mathrm{dad}\right)\right]$ complexes.}

Table S5. FOD and MOS analysis of various [M(trop2dad)] complexes.

\begin{tabular}{|l|l|l|l|l|}
\hline CSD ID & MF $\left(\mathrm{L}=\right.$ trop $_{2}$ dad $)$ & $\mathrm{N}_{\mathrm{FOD}}$ & $\mathrm{N}^{\mathrm{R}} \mathrm{FOD}^{a}$ & $\mathrm{MOS}$ \\
\hline HEVWOW & {$[\mathrm{Ru}(\mathrm{L}) \mathrm{H}]^{-}$} & 0.78 & 0.31 & -1.22 \\
\hline YARYAU & {$[\mathrm{Ru}(\mathrm{L}) \mathrm{H}]^{-}$} & 0.83 & 0.33 & -1.35 \\
\hline YASMUD & {$[\mathrm{Ru}(\mathrm{L}) \mathrm{H}]^{-}$} & 0.78 & 0.32 & -1.29 \\
\hline IKUFOJ & {$[\mathrm{Rh}(\mathrm{L})]^{+}$} & 0.90 & 0.36 & -0.24 \\
\hline HEVXIR & {$\left[\mathrm{Ru}(\mathrm{L})\left(\mathrm{PPh}_{3}\right)\right]$} & 0.87 & 0.23 & -1.16 \\
\hline HEZXIW & {$[\mathrm{Ru}(\mathrm{L})(\mathrm{thf})]$} & 0.97 & 0.34 & -0.91 \\
\hline JUXPAV & {$\left[\mathrm{Fe}(\mathrm{L})\left(\mathrm{CH}{ }_{3} \mathrm{CN}\right)\right]$} & 1.02 & 0.37 & -0.97 \\
\hline JUXPEZ & {$\left[\mathrm{Fe}(\mathrm{L})\left(\mathrm{PPh}_{3}\right)\right]$} & 1.12 & 0.28 & -0.96 \\
\hline JUXPID & {$\left[\mathrm{Fe}(\mathrm{L})\left(\mathrm{P}(\mathrm{OMe}){ }_{3}\right)\right]$} & 0.82 & 0.26 & -0.97 \\
\hline
\end{tabular}

${ }^{a} \mathrm{~N}^{\mathrm{R}} \mathrm{FOD}=100 * \mathrm{~N}_{\mathrm{FOD}} /$ (number of electrons).

\section{S10 FOD plots of $[M($ trop 2 dad $)]$ complexes.}

Electron density plots obtained from FOD analysis for complexes listed in Table S3, $\left(\rho=0.005\right.$ e $\left.\mathrm{Bohr}^{-3}\right)$.

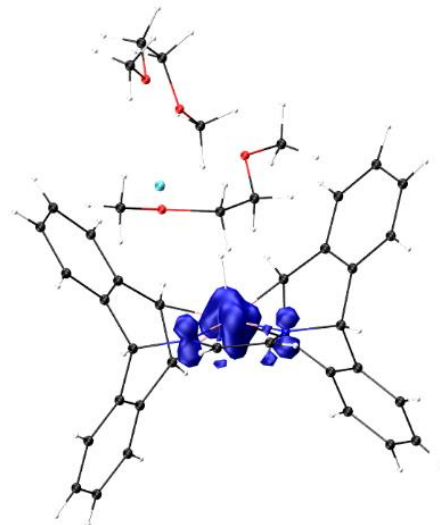

Figure S5. HEVWOW 


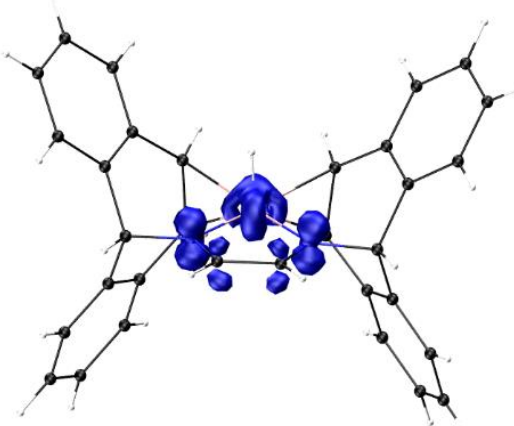

Figure S6. YARYAU

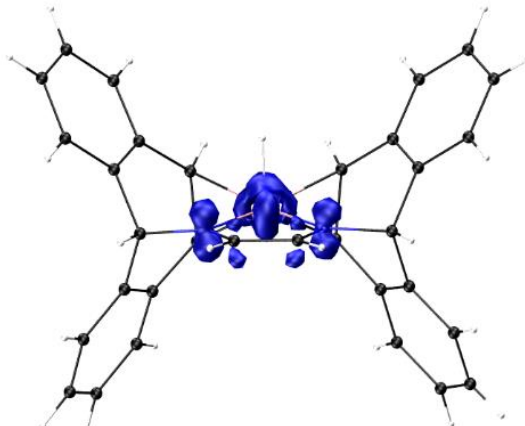

Figure S7. YASMUD

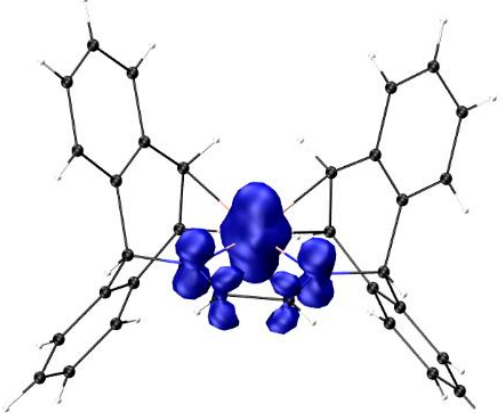

Figure S8. IKUFOJ 


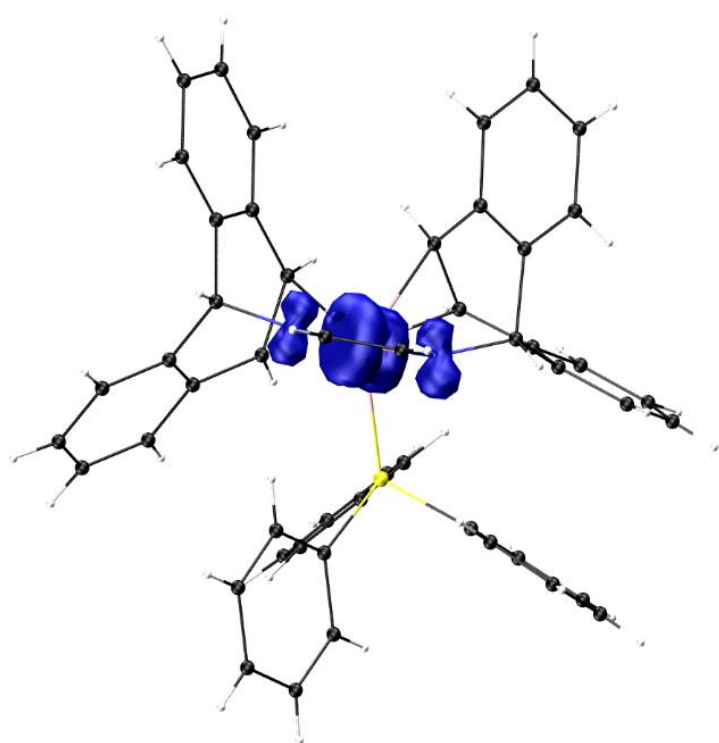

Figure S9. HEVXIR

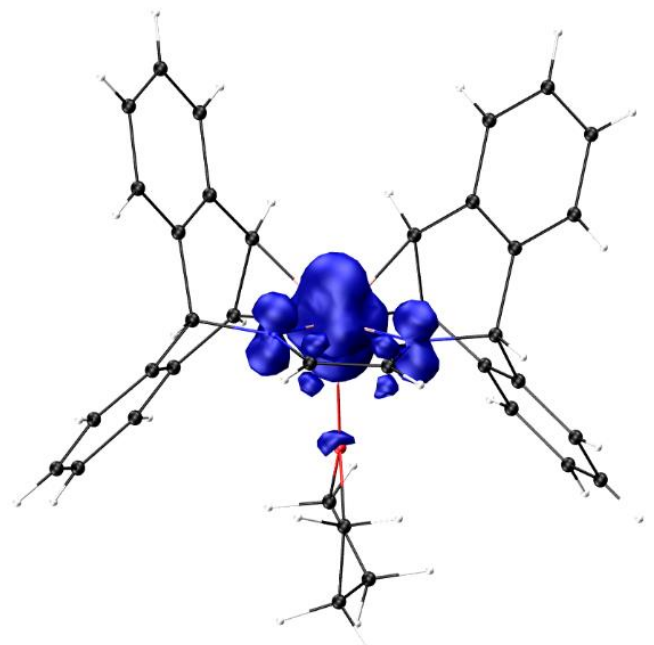

Figure S10. HEZXIW 


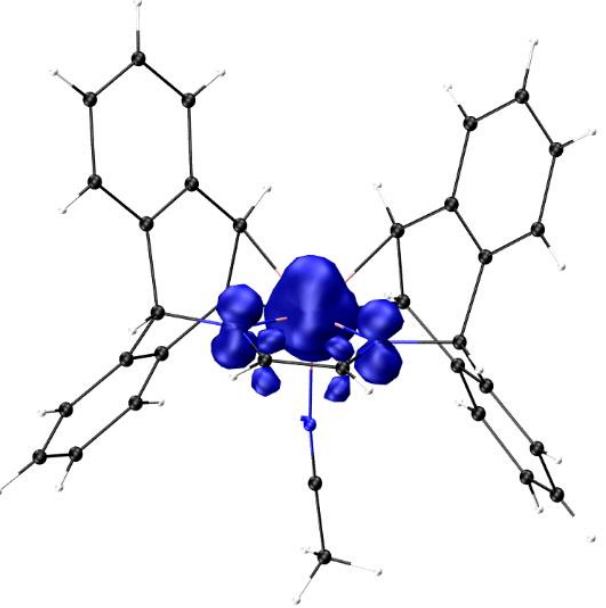

Figure S11. JUXPAV

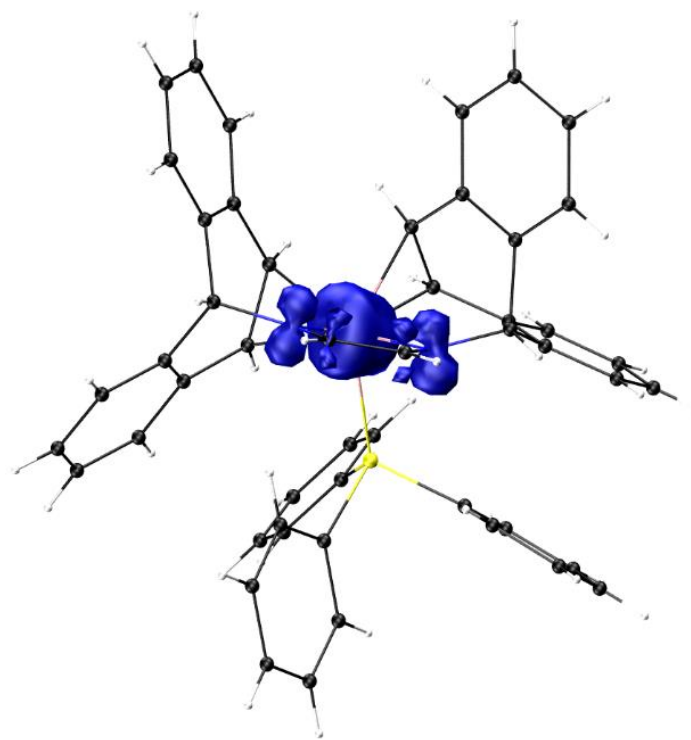

Figure S12. JUXPEZ 


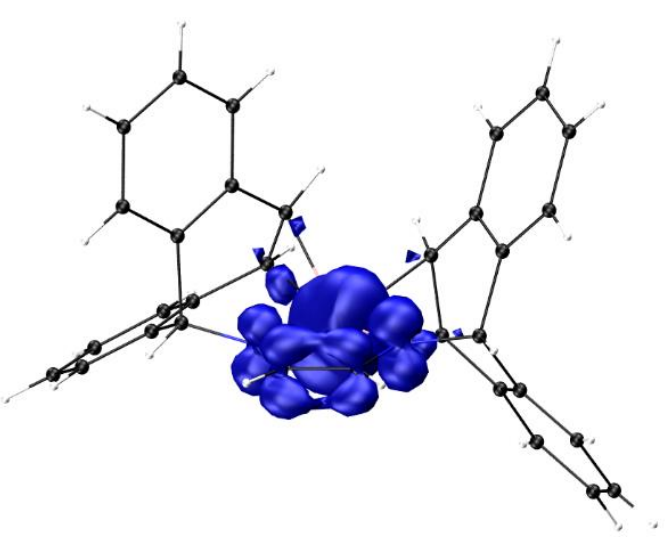

Figure S13. JUXPID

\section{References in the Supporting Information}

(1) Olsen J. The CASSCF Method: A Perspective and Commentary, Int. J. of Quantum Chem., 2011, 111, 3267-3272.

(2) Neese, F. The ORCA program system, WIREs Comput. Mol. Sci., 2012, 2, 73-78.

(3) Grimme, S.; Hansen, A. A Practicable Real-Space Measure and Visualization of Static Electron-Correlation Effects, Angew. Chem. Int. Ed., 2015, 54, 12308-12313.

(4) Tao, J.; Perdew, J.P.; Staroverov, V.N.; Scuseria, G.E. Climbing the density functional ladder: Nonempirical meta-generalized gradient approximation designed for molecules and solids, Phys. Rev. Lett. 2003, 91, 146401.

(5) (a) Schäfer, A.; Horn, H.; Ahlrichs, R. Fully optimized contracted Gaussian basis sets for atoms Li to Kr, J. Chem. Phys. 1992, 97, 2571. (b) Weigend, F.; Ahlrichs, R. Balanced basis sets of split valence, triple zeta valence and quadruple zeta valence quality for H to Rn: Design and assessment of accuracy, Phys. Chem. Chem. Phys. 2005, 7, 3297.

(6) Bauer, C.A.; Hansen, A.; Grimme, S. The fractional occupation number weighted density as a versatile analysis tool for molecules with a complicated electronic structure, Chem. - A Eur. J., 2017, 23, 6150-6164.

(7) Sinha, V.; Govindarajan, N.; De Bruin, B.; Meijer, E.J. How Solvent Affects C-H Activation and Hydrogen Production Pathways in Homogeneous Ru-Catalyzed Methanol Dehydrogenation Reactions ACS Catalysis, 2018, 8, 6908-6913. 